\title{
Monte Carlo Simulations of the ISS-CREAM Instrument
}

\author{
J. Wu' ${ }^{1 a}$, Y. Amare ${ }^{a}$, D. Angelaszek ${ }^{a, b}$, N. Anthony ${ }^{a}$, G. H. Choi ${ }^{c}$, M. Chung $^{a}$, M. \\ Copley $^{a}$, L. Derome ${ }^{d}$, L. Eraud ${ }^{d}$, C. Falana ${ }^{a}$, A. Gerretya, L. Hagenau ${ }^{a}$, J. H. Han ${ }^{a}$, H. \\ G. Huh ${ }^{a}$, Y. S. Hwang ${ }^{e}$, H. J. Hyun ${ }^{e}$, H.B. Jeon ${ }^{e}$, J. A. Jeon', S. Jeong', S. C. Kang, ${ }^{\text {e }}$

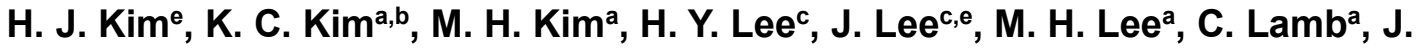 \\ Liang $^{\mathrm{a}}$, L. Lu ${ }^{\mathrm{a}}$, J. P. Lundquist ${ }^{\mathrm{a}, \mathrm{c}}$, L. Lutz ${ }^{\mathrm{a}}$, B. Mark ${ }^{\mathrm{a}}$, A. Menchaca-Rocha ${ }^{\mathrm{f}}$, T. \\ Mernik $^{\text {a }}$, M. Nester ${ }^{\mathrm{a}}$, O. Ofoha ${ }^{\mathrm{a}}$, H. Park ${ }^{\mathrm{e}}$, I. H. Park ${ }^{\mathrm{c}}$, J. M. Park ${ }^{\mathrm{e}}$, N. Picot- \\ Clemente $^{a}$, S. Rostskya ${ }^{\text {a }}$ E. S. Seo ${ }^{a, b}$, J. R. Smith ${ }^{a}$, R. Takeishic, T. Tatolia, P. \\ Walpole $^{\mathrm{a}}$, R. P. Weinmann ${ }^{\mathrm{a}}$, Z. Yin ${ }^{\mathrm{a}, \mathrm{b}}$, Y. S. Yoon ${ }^{\mathrm{a}, \mathrm{b}}$ and H. G. Zhang ${ }^{\mathrm{a}}$ \\ ${ }^{a}$ Inst. for Phys. Sci. and Tech., University of Maryland, College Park, MD, USA \\ ${ }^{b}$ Dept. of Physics, University of Maryland, College Park, MD, USA \\ ${ }^{c}$ Dept. of Physics, Sungkyunkwan University, Republic of Korea \\ ${ }^{d}$ Laboratoire de Physique Subatomique et de Cosmologie, Grenoble, France \\ ${ }^{e}$ Dept. of Physics, Kyungpook National University, Republic of Korea \\ finstituto de Fisica, Universidad Nacional Autonoma de Mexico, Mexico
}

\begin{abstract}
Cosmic Ray Energetics and Mass for the International Space Station (ISS-CREAM) is designed to directly measure the energy spectra of high-energy cosmic rays, encompassing proton to iron nuclei, over the energy range from $10^{12}$ to $10^{15} \mathrm{eV}$ [1]. The capability to measure an extended energy range enables us to probe the origin and acceleration mechanisms of cosmic rays. The ISSCREAM instrument is configured with the balloon-borne CREAM calorimeter (CAL) for energy measurements and four layers of a finely segmented Silicon Charge Detector (SCD) for charge measurements. In addition, two new compact detectors have been developed for electron/proton separation: Top and Bottom scintillator-based counting detectors (TCD/BCD) and a boronated scintillator detector (BSD). Simulations use the GEANT3 package [2] with the FLUKA hadronic model [3]. An isotropic event generator was developed for the ISS-CREAM geometry with particles incident from the upper hemisphere. We will present simulation results regarding ISSCREAM performance, including trigger rates, energy resolution, energy response, tracking resolution, charge efficiency, etc.
\end{abstract}

36th International Cosmic Ray Conference -ICRC2019-

July 24th - August 1st, 2019

Madison, WI, U.S.A.

\footnotetext{
${ }^{1}$ Speaker for the ISS-CREAM collaboration

E-mail: jayoung@umd.edu
}

For collaboration list see PoS(ICRC2019)1177 


\section{Introduction}

The ISS-CREAM experiment is the successor of the Antarctic balloon-borne CREAM mission, which flew successfully seven times for a total accumulated time of 191 days. The ISSCREAM instrument was successfully installed, and activated, in the ISS Japanese Experiment Module External Facility 2 (JEM-EF 2) on August 22, 2017. Its long exposure above the atmosphere offers orders of magnitude greater statistics without the secondary particle background inherent in balloon experiments investigating the origin of cosmic rays. To study its performance we have developed a Monte Carlo simulation model of the instrument.

\section{ISS-CREAM Instrument}

The ISS-CREAM instrument consists of complementary and redundant particle detectors (Figure 1). An ionization calorimeter determines the energy of cosmic ray particles, provides tracking, and a high energy trigger. Carbon-Targets induce hadronic interactions. The four-layer Silicon charge detectors (SCD) provide precise charge measurements. Top/bottom counting detectors $(\mathrm{TCD} / \mathrm{BCD})$ provide shower profiles for electron/hadron separation and a lower energy trigger. The boronated scintillator detector (BSD) provides additional electron/hadron discrimination using thermal neutrons produced by particles that interact within the calorimeter. For a detailed description of ISS-CREAM instrument configuration, refer to [4].

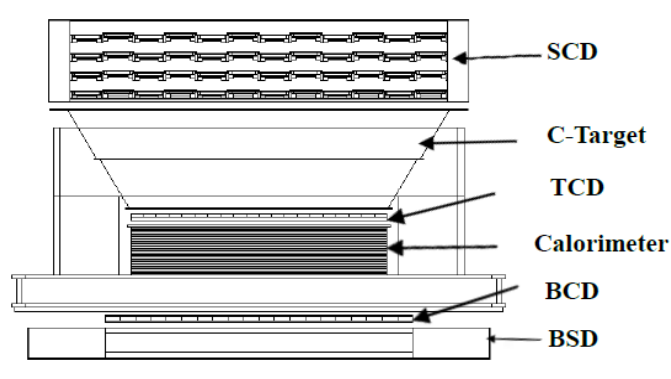

Figure 1. ISS-CREAM detector configuration in simulations

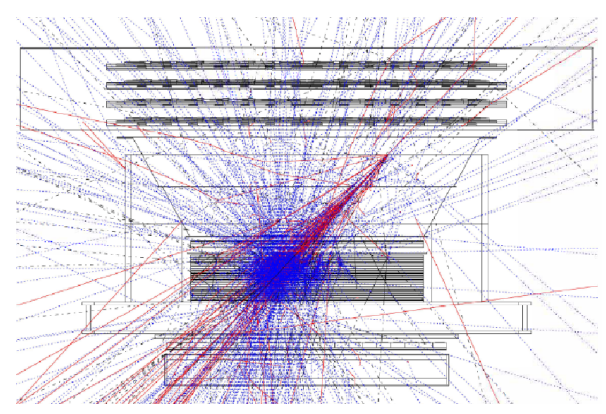

Figure 2. An example of simulated event: gamma (blue), charged particles except muons (red), neutral hadrons or neutrinos (black)

\section{Monte Carlo Simulations}

Our simulation model is based on the GEANT Monte Carlo simulation toolkit, which is widely used for various collider experiments. GEANT simulates the passage of particles through matter, allowing for arbitrary geometry, materials, and magnetic field configuration. It accounts for particle decays and interactions, with subsequent tracking of all secondary particles. GEANT includes the following processes: pair creation, Compton scattering, photoelectric effect, photo fission, Rayleigh scattering, multiple scattering, ionization, delta-ray production, bremsstrahlung, positron annihilation, Moliere scattering, Cherenkov light generation, synchrotron radiation, particle decays, and more. We have tested several hadron interaction packages and chosen FLUKA as the baseline for our simulation model [5]. FLUKA is based on the dual parton model for hadron interactions, so it is expected to provide better simulation at higher energies, say $\mathrm{E}>$ $10 \mathrm{TeV}$. In this study protons have been generated isotropically over an incident energy range from $10 \mathrm{GeV}$ to $200 \mathrm{TeV}$. A typical event passes first through the charge module, then interacts in the carbon target, and finally develops a shower in the calorimeter (Figure 2). 


\section{Analysis}

\subsection{Event selection}

The first level of event selection is by the trigger on the calorimeter response. An event is triggered when an incident cosmic ray deposits energy greater than $76 \mathrm{MeV}$ on one fiber ribbon or more through at least six consecutive layers of the calorimeter. High-energy shower events that meet this calorimeter trigger condition were used in this analysis. The fraction of triggered events is about $75 \%$ of in geometry (Ingeo) events above couple of $\mathrm{TeV}$ while the fraction of good events is about 54\%, as shown in Figure 3. Ingeo events are events in which the particles enter charge module(SCD), and pass through all the layers of the calorimeter. The good events were obtained by selecting events that have their first interaction anywhere in the carbon target among Ingeo events. Error bars are smaller than the symbol size on the plot.

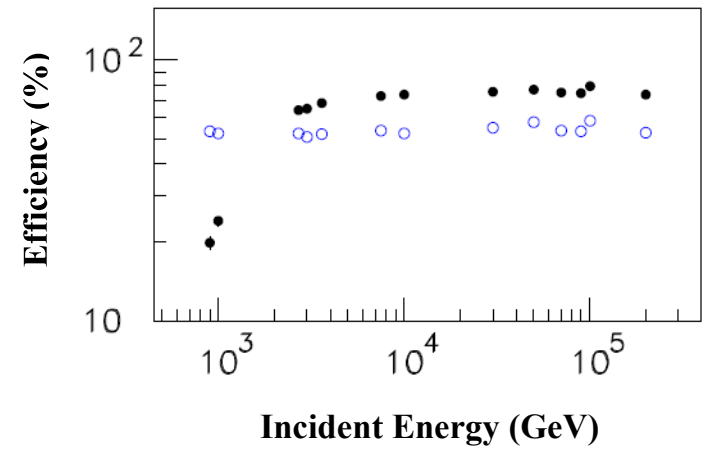

Figure 3: Incident energy dependence of event selection; triggered events (filled circle), good events. (open circle)

\subsection{Calorimeter energy response}

The mean energy deposit in calorimeter and the energy resolution are shown as a function of incident energy in Figures 4 and 5, respectively. The mean energy deposit is quite linear with

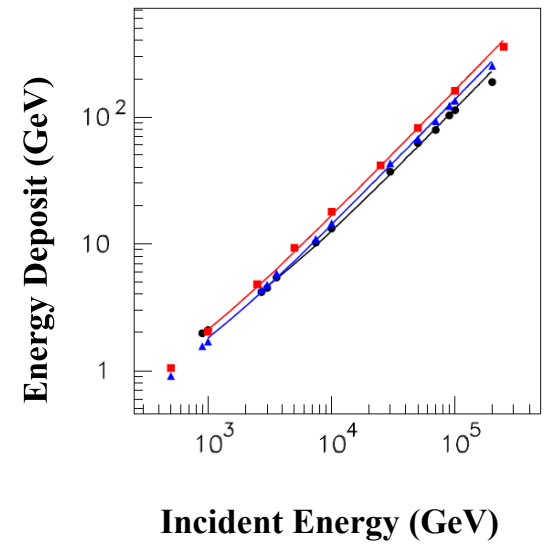

Figure 4: Incident energy dependence of the mean energy deposit in calorimeter for protons; triggered events (black circles), good events (blue triangles), good events with CREAM-III (red rectangles), linear fit (lines).

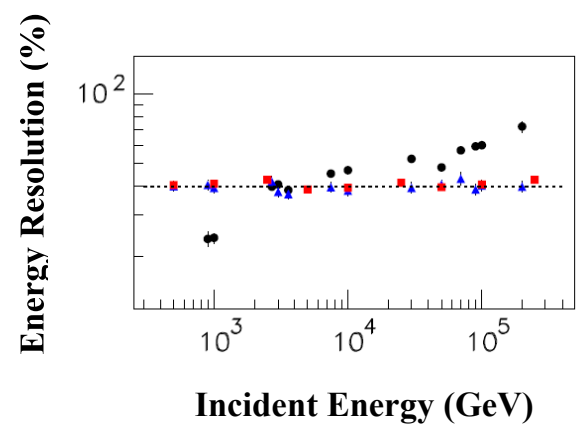

Figure 5: Incident energy dependence of the energy resolution for protons; triggered events (black circles), good events (blue triangles), good events with CREAM-III (red rectangles). 
the incident energy. The slopes of linear fit are $1.14 \times 10^{-3}, 1.37 \times 10^{-3}, 1.62 \times 10^{-3}$ for triggered events, good events, and good events with CREAM-III, respectively. The energy resolution, about $40 \%$ for the good events, is quite independent of the incident energy. The results with good events are compared with a balloon-borne CREAM experiment, CREAM-III. The mean energy deposit with ISS-CREAM is slightly lower than with CREAM-III.

Figure 6 shows the mean energy deposit in each layer as a function of the calorimeter depth. The longitudinal shower profiles for proton good events are presented. The incident energy ranges from $100 \mathrm{GeV}$ at the bottom to $100 \mathrm{TeV}$ at the top. The shower starts at the top layer, reaches its maximum in the middle layers, and then dies down. The higher the energy, the deeper the shower maximum is located in the calorimeter. Even the highest energy shower maxima are well contained.

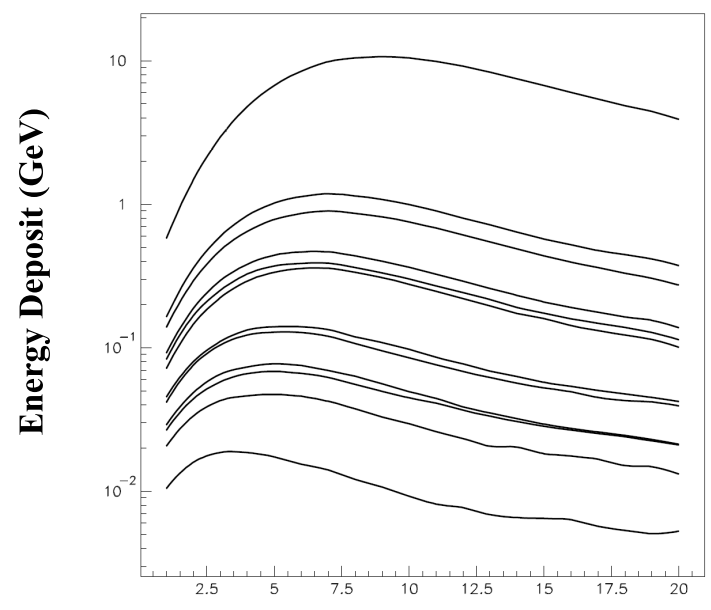

Layer Number

Figure 6: Longitudinal shower profile for proton good events. From the bottom, incident energy is $100 \mathrm{GeV}$, $300 \mathrm{GeV}, 450 \mathrm{GeV}, 500 \mathrm{GeV}, 900 \mathrm{GeV}, 1 \mathrm{TeV}, 2.7 \mathrm{TeV}, 3 \mathrm{TeV}, 3.6 \mathrm{TeV}, 7.5 \mathrm{TeV}, 10 \mathrm{TeV}$, and $100 \mathrm{TeV}$.

\subsection{Shower axis reconstruction algorithm}

Due to the backscattering of secondaries, a large fraction of background events triggers the system. To remove theses background events, we have to reconstruct the incident particle trajectory. A tracking algorithm is used to reconstruct the particle trajectory by calculating the energy deposit centroid in each calorimeter layer, thereby providing up to $10 \mathrm{x}$ and y cascade coordinate pairs. The cascade axis is determined by linear fitting of these coordinates separately in $\mathrm{x}$ and in $\mathrm{y}$. In order to reduce fluctuations, two requirements are applied on the tracking algorithm: (1) each calorimeter layer used in the fitting is required to have an energy deposit greater than $3 \%$ of the total energy deposit in the entire calorimeter. (2) an event is accepted if it has at least 3 calorimeter layers that satisfied the above condition in $\mathrm{x}$ and $\mathrm{y}$ in each direction: otherwise, it is rejected.

\subsection{Trajectory resolution}

By extrapolating the reconstructed trajectory to the charge detector(SCD), the entrance position of the primary particle is calculated. The deviation between the actual incident position and this reconstructed position is a Gaussian distribution with a sigma (position resolution) of about 
1 pixel as shown in Figure 7; for $\mathrm{x}$ direction (left), and for y direction (right). Dotted lines represent SCD pixel width (left) and length (right). The position resolution at the top of the calorimeter is $0.2 \mathrm{~cm}$ which is better than on the SCDs due to extrapolating to a shorter distance.
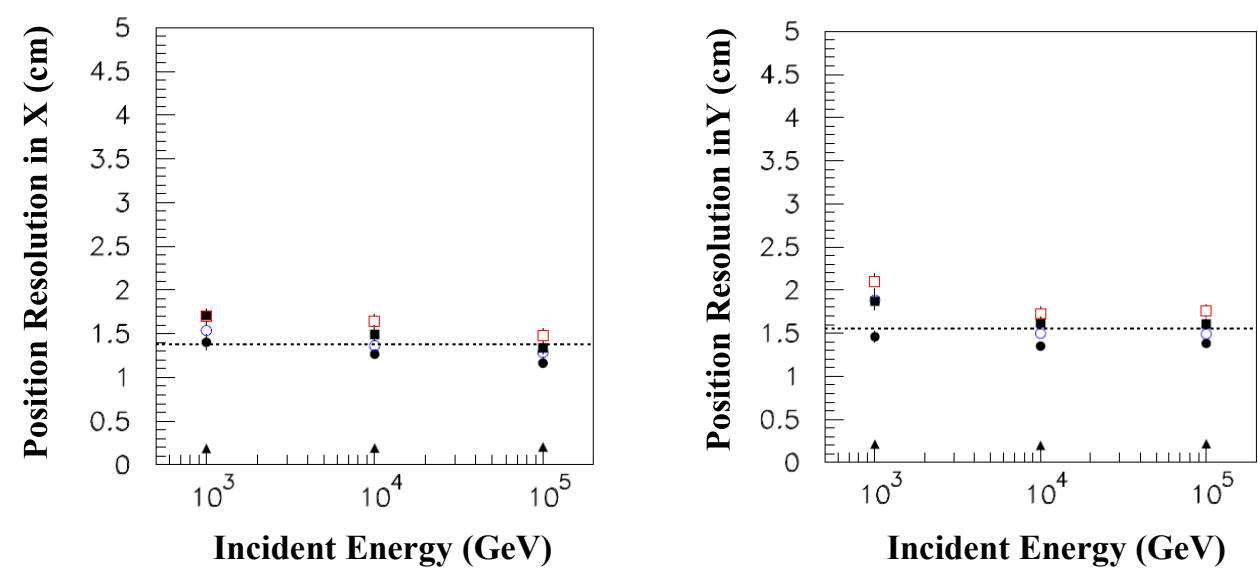

Figure 7: Incident energy dependence of the position resolution for protons; from the top SCD1 (open red squares), SCD2 (filled black squares), SCD3 (open blue circles), SCD4 (filled black cicles), and Calorimeter (filled black triangles)

\subsection{Charge measurement}

\subsubsection{Charge determination and charge resolution}

In order to determine the incident particle charge, the reconstructed shower axis from the calorimeter was extrapolated to the SCD and a $7 \times 7$ pixel area of circle of confusion, about $10 \times$ $10 \mathrm{~cm}^{2}$, centered on the extrapolated position, was scanned to seek for the highest pixel signal [6]. If no signal is found, the event is considered not to satisfy charge requirement and is rejected. That highest pixel signal was then corrected for the particle path length (calculated from the reconstructed incidence angle) of the pixel. The signal reflects the ionization energy loss per unit path length $(\mathrm{dE} / \mathrm{dx})$ of an incident particle in the SCD. The energy loss is proportional to $Z^{2}$. The resulting SCD signal distribution is shown in Figure 8. Events with $Z<1.732$ were identified as protons, while events with $1.732 \leq \mathrm{Z}<2.739$ were misidentified as helium nuclei.

\subsubsection{Charge efficiency and backscattering}

Charge efficiency, the fraction of events that satisfy the charge requirement condition among the reconstructed events that traverse SCD active area, is 90 95\% depending on SCD layers. The identified proton fraction is 74 97\% depending on SCD layers and incident energy, as shown in Table 2. The identified proton fraction is ratio to the selected events that pass the charge requirement condition. The fraction decreases in deeper SCDs and at higher incident energies due to the backscattering effect. It is characteristic of a calorimeter that backscattered particles can reach the charge detector and confuse the charge measurement. The number of backscattered particles increases with the incident energy, so the probability for protons to mimic helium or higher charge particles increases. 

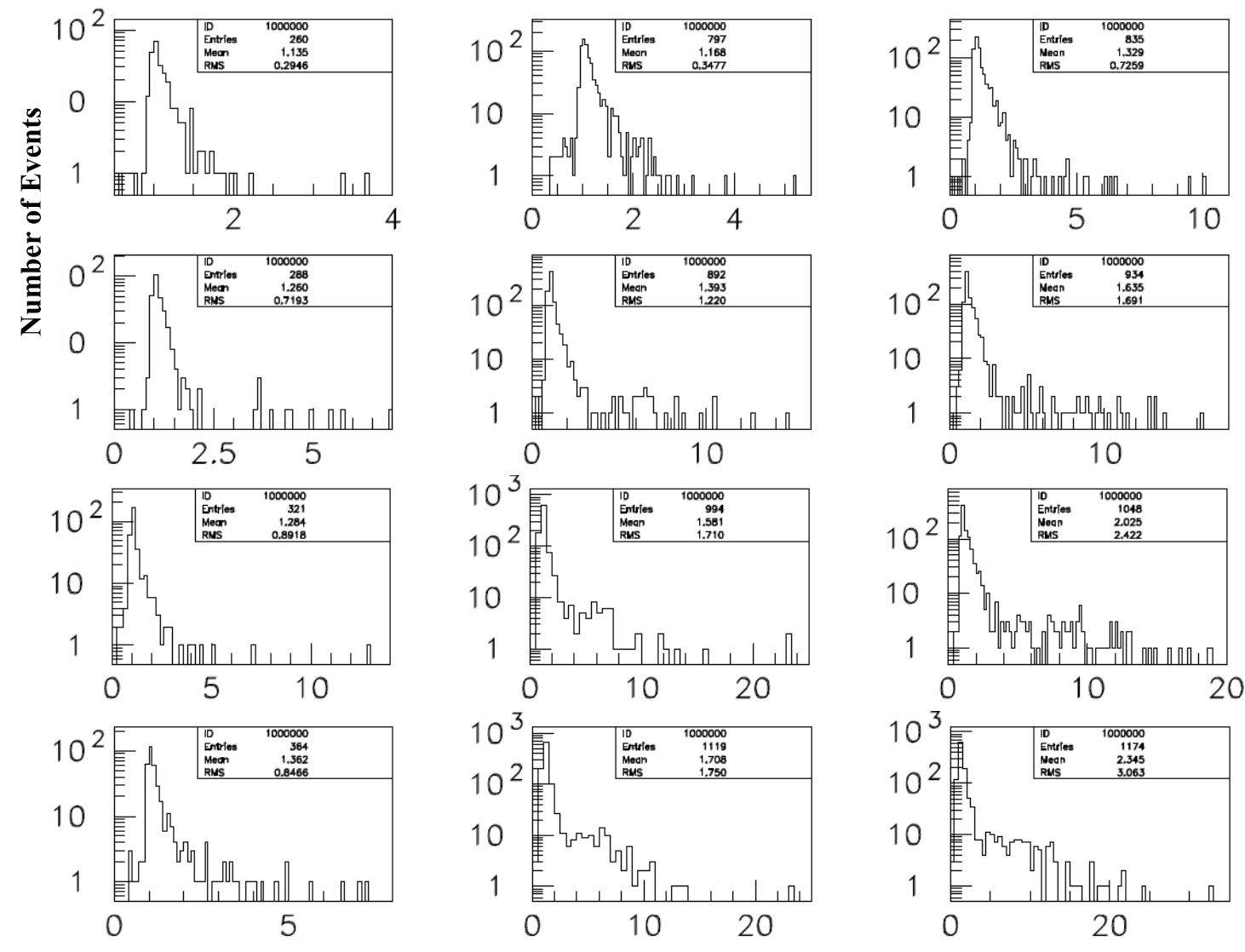

SCD Charge (Z)

Figure 8: Charge distribution of selected events with the SCD for protons. From the top to bottom is with SCD1, SCD2, SCD3, and SCD4. From left to right, incident energy is $1 \mathrm{TeV}, 10 \mathrm{TeV}$, and $100 \mathrm{TeV}$.

Table 1: Charge efficiency and identified proton fraction for isotropic protons: incident energy $1 \mathrm{TeV}, 10 \mathrm{TeV}$, and $100 \mathrm{TeV}$

\begin{tabular}{|l|l|l|l|}
\hline Energy & $\mathbf{1 ~ T e V}$ & $\mathbf{1 0} \mathbf{~ T e V}$ & $\mathbf{1 0 0} \mathbf{~ T e V}$ \\
\hline Triggered events & 479 & 1890 & 2218 \\
\hline Track Reconstr. in SCD1 & $291(60.8 \%)$ & $887(46.9 \%)$ & $914(41.2 \%)$ \\
Track Reconstr. in SCD2 & $312(65.1 \%)$ & $979(51.8 \%)$ & $1010(45.5 . \%)$ \\
Track Reconstr. in SCD3 & $350(73.1 \%)$ & $1079(57.1 \%)$ & $1113(50.2 \%)$ \\
Track Reconstr. in SCD4 & $386(80.6 \%)$ & $1189(62.9 \%)$ & $1238(55.8 \%)$ \\
\hline$Z>0$ in SCD1 & $260(89.3 \%)$ & $797(89.9 \%)$ & $835(91.4 \%)$ \\
$Z>0$ in SCD2 & $288(92.3 \%)$ & $892(91.1 \%)$ & $934(92.5 \%)$ \\
$Z>0$ in SCD3 & $321(91.7 \%)$ & $994(92.1 \%)$ & $1048(94.2 \%)$ \\
$Z>0$ in SCD4 & $364(94.3 \%)$ & $1119(94.1 \%)$ & $1174(94.8 \%)$ \\
\hline Identified p in SCD1 & $252(96.9 \%)$ & $755(94.7 \%)$ & $740(88.6 \%)$ \\
Identified p in SCD2 & $270(93.8 \%)$ & $808(90.6 \%)$ & $788(84.4 \%)$ \\
Identified p in SCD3 & $291(90.7 \%)$ & $876(88.1 \%)$ & $815(77.8 \%)$ \\
Identified p in SCD4 & $318(87.4 \%)$ & $934(83.5 \%)$ & $863(73.5 \%)$ \\
\hline
\end{tabular}




\section{Summary}

We have developed GEANT-based simulation models of the ISS-CREAM instrument to study its performance. In this paper, we presented simulation results for protons. The energy dependence of the mean energy deposition is robustly linear over the entire simulated energy range. The energy resolution, about $40 \%$ for good events, is quite independent of the incident energy.

Further studies require extending simulation of the detector response to $Z>1$ cosmic rays. Since GEANT does not accommodate the simulation of heavy ions, we have interfaced GEANT with hadronic simulation packages for heavy ion interactions; a nucleus-nucleus interaction event generator based on FRITIOF is used for high energies, while RQMD and DPMJET are used for low energies [7]. These nucleus-nucleus interaction codes are used to create the first interaction, whose secondary hadrons are then fed into the GEANT simulation routine. Simulation runs with heavy ions are ongoing.

\section{Acknowledgements}

This work was supported in the U.S. by NASA grant NNX17AB41G, in Korea by National Research Foundation grants 2018R1A2A1A05022685 and 2018R1A6A1A06024970, and their predecessor grants. It was also supported in France by IN2P3/CNRS and CNES and in Mexico by DGAPA-UNAM project IN109617. The authors thank NASA GSFC WFF and its contractors for engineering support and project management, JSC ISS Program Office for the launch support and ISS accommodation, MSFC for the operational support, and KSC and SpaceX for the launch support.

\section{References}

[1] E.S. Seo, et al., Adv. in Space Res, 53/10, 1451-1455, 2014.

[2] R. Brun, et al., GEANT User's Guide, CERN, DD/EE/84-1, 1984.

[3] P.A. Arino, et al., FLUKA User's Guide, CERN, TIS-RP-190, 1987

[4] J.R. Smith, et al, Proc. 35th Int. Cosmic Ray Conf., PoS(ICRC2017)199.

[5] E.S. Seo, et al., SPIE Proc., 2806, 134-144, 1996.

[6] Y.S. Yoon, et al., The Astrophys. J., 728, 122, 2011.

[7] H.J. Kim, et al. Proc. 26th Int. Cosmic Ray Conf., 1, 17-20, 1999 